\title{
Impact of vitamin $D$ serum levels on clinicopathological features and outcome in advanced pancreatic carcinoma
}

Research Article

\author{
Amrallah A. Mohammed ${ }^{1,2 *}$, Reham A. Salem ${ }^{3,4}$ \\ ${ }^{1}$ Medical Oncology Department, Faculty of Medicine, \\ Zagazig University, Egypt \\ 2King Salman Armed Forces Hospital-Tabuk City, KSA \\ ${ }^{3}$ Department of clinical oncology and nuclear medicine, \\ Faculty of medicine Zgazig University \\ ${ }^{4}$ Department of Radiation Therapy Princess Nourah \\ bint Abdulrhman University
}

Received 17 March 2019; Accepted 11 December 2019

\begin{abstract}
Background: Growing evidence encourages the preventive role of vitamin D in pancreatic carcinoma (PC). Meanwhile, the prognostic or predictive role needs more investigations. This study aimed to evaluate the correlation between serum vitamin $D$ levels and the clinicopathological features with the outcome in advanced pancreatic carcinoma (APC).

Materials and methods: The current prospective study included 176 patients with APC. Assessing 25-hydroxy vitamin D is the most accurate method to measure the serum vitamin D levels. Serum vitamin D levels $<20 \mathrm{ng} / \mathrm{ml}$ are defined as vitamin D deficiency, while levels ranging from 20 to $29 \mathrm{ng} / \mathrm{ml}$ are defined as vitamin $D$ insufficiency. Before any treatment modalities were administered, the serum vitamin D levels were measured by enzyme-linked immunosorbent assay (ELISA).

Results: Serum vitamin D insufficiency and deficiency were detected in $28.4 \%$ and $31.8 \%$, respectively. A large tumor size, higher grade, liver metastasis, higher serum level of CA 19-9, poor ECOG PS, and low overall response rate (ORR) were associated with lower serum vitamin $\mathrm{D}$ levels $(p=0.000)$. The median follow-up period was 7.6 months (range $0.6-18.6$ ). The ORR was $23.2 \%, 54 \%$, and $82.9 \%$ of vitamin $D$ deficiency, insufficiency, and normal levels of vitamin $D$, respectively. The median OS was 11.4 months for patients with normal serum vitamin D levels, compared with 2.7 and 7.03 months for serum vitamin D deficiency and insufficiency, respectively. Conclusions: Among patients with APC, serum vitamin D levels are considered a promising prognostic factor. It is associated with various poor prognostic features and worse survival outcome.
\end{abstract}

Keywords: Vitamin D level • advanced pancreatic carcinoma • overall survival

\section{Introduction}

Around 55,440 new cases of pancreatic carcinoma (PC) were diagnosed in the United States with 44,330 deaths in 2018. A stage for stage PC is associated with the lowest survival rates compared with any other major type of cancer ${ }^{[1]}$. By 2030, it is expected to rise to the next position after lung cancer as a major cause of cancer-related death ${ }^{[2]}$. Unfortunately, most of the patients (>80\%) present in an advanced stage owing to the nonspecific symptoms or signs ${ }^{[3]}$. So, any attempt to reduce the dismal outcome of this fatal cancer will attract public health support.

The relation between serum vitamin $D$ levels and health status is a dynamic process. Voluminous studies had reported the link between vitamin $D$ serum levels and several types of cancers such as colorectal cancer, breast cancer, and PC. These findings pushed the investigators to evaluate the value of serum vitamin $D$ testing as well as supplementation with an aim to detect and treat the low levels ${ }^{[4,5]}$. 
Vitamin D is a group of fat-soluble vitamins; it acts as a hormone which is responsible for the absorption of many minerals including calcium to maintain the normal calcium level and prevent many degenerative diseases, as well as has a postulated role in cancer. Vitamin D3, also called cholecalciferol, is the most common one. The interaction between sunlight and the skin represents the main source of vitamin D. So, during winter, the use of fortified food must be considered. Hypovitaminosis D is mainly due to insufficient sunlight exposure, and it affects about $50 \%$ of the population worldwide ${ }^{[6,7]}$.

In PC, the vitamin D receptor (VDR) is overexpressed in human PC cell lines, compared with normal pancreatic cells. Early trials reported the importance of vitamin $\mathrm{D}$ metabolism in maintaining the PC. However, Waterhouse et al. contradicted the presence of a relationship between dietary vitamin $D$ intake and PC prevention. So far, randomized control trials are insufficient and without reliable outcome ${ }^{[8-11]}$.

Based on different geographical, laboratory, observational studies, as well as clinical trials, Grant reported a possible role of vitamin $D$ in cancer ${ }^{[12]}$. However, the data are more complex and more effort is needed to clarify the actual role of vitamin D in PC, either prognostic, therapeutic, or protective.

Therefore, in the current study, we aimed to investigate the correlation between serum vitamin $D$ levels and the clinicopathological features with the survival outcome in advanced pancreatic carcinoma (APC).

\section{Materials and Methods}

The current prospective study included 183 APC patients during the period from March 2016 to January 2018 who were investigated and treated in the Medical Oncology Department, Faculty of Medicine, Zagazig University and El-Mabra Hospital. The eligibility criteria were: pathologically proved diagnosis of PC, evidence of advanced stage disease, chemotherapy-naïve disease, and age $\geq 18$ years. The exclusion criteria were: early cases of the adjuvant setting or advanced cases after multiple lines of treatment. The demographic data, clinicopathological features, and survival outcome were recorded through clinical examination and from the medical files. The staging was done according to the American Joint Committee on Cancer (AJCC). Treatment choice was based on the local institutional guidelines.

\subsection{Assessment of serum 25 -hydroxy vitamin D levels}

Serum 25-hydroxy vitamin $\mathrm{D}(25(\mathrm{OH}) \mathrm{D})$ levels below 20 $\mathrm{ng} / \mathrm{ml}$ and ranging from 20 to $29 \mathrm{ng} / \mathrm{ml}$ are defined as vitamin $D$ deficiency and insufficiency, respectively, while a serum level $\geq 30 \mathrm{ng} / \mathrm{ml}$ is considered to be normal[13]. Following manufacturer's instructions, vitamin D was measured by the enzyme-linked immunosorbent assay (ELISA) technique before starting any line of treatment in eligible patients.

\subsection{Statistical analysis}

Continuous variables were expressed as the mean \pm SD and median (range), and the categorical variables were expressed as a number (percentage). Continuous variables were checked for normality by using the Shapiro-Wilk test. Percentage of categorical variables was compared using Pearson's Chi-square test or Fisher's exact test, whichever was appropriate. The Kruskal-Wallis test was used to compare more than two groups of non-normally distributed variables. Overall survival (OS) was calculated as the time from diagnosis to death or the most recent follow-up contact (censored). Stratification of OS was done according to the vitamin D3 level. These time-to-event distributions were estimated using the method of Kaplan-Meier plot and compared using two-sided exact log-rank test. Cox regression analysis was performed to study the relationship between different study variables as independent predictors and mortality as an outcome or a dependent variable. A $p$-value $<0.05$ was considered significant. All statistics were performed using SPSS 20.0 for Windows (SPSS Inc., Chicago, IL, USA) and MedCalc windows (MedCalc Software bvba 13, Ostend, Belgium).

\section{Results}

\subsection{Clinicopathological features of 176 PC patients}

A total of 183 patients were eligible; 7 patients were excluded due to insufficient data. The median age was 61 years (range 30-80), and approximately 60\% of the patients were male. The most common sites of metastasis were liver $(76.7 \%)$, peritoneum $(61.9 \%)$, and lung $(30.7 \%)$. The median serum level of CA 19-9 was 698 (range $21.00-78,962.00) \mathrm{IU} / \mathrm{ml}$. Lower serum 
Table 1: Clinicopathological features among 176 advanced pancreatic carcinoma patients.

\begin{tabular}{|c|c|c|c|c|c|}
\hline \multirow{3}{*}{ Characteristics } & \multicolumn{2}{|c|}{ All } & \multirow{3}{*}{ Characteristics } & \multirow{2}{*}{\multicolumn{2}{|c|}{$\begin{array}{c}\text { All } \\
(N=176)\end{array}$}} \\
\hline & \multicolumn{2}{|c|}{$(N=176)$} & & & \\
\hline & No. & $\%$ & & No. & $\%$ \\
\hline Age (years) & \multirow{2}{*}{\multicolumn{2}{|c|}{$60.92 \pm 7.79$}} & ECOG PS & 10 & 57 \\
\hline Mean \pm SD & & & ECOG 0 & 10 & 5.7 \\
\hline Median (Range) & \multicolumn{2}{|c|}{$61(30-80)$} & ECOG 1 & 72 & 40.9 \\
\hline Sex & & & ECOG 2 & 64 & 36.4 \\
\hline Male & 105 & 59.7 & ECOG 3 & 23 & 13.1 \\
\hline Female & 71 & 40.3 & ECOG 4 & 7 & 4 \\
\hline \multirow[t]{2}{*}{ Grade } & \multicolumn{5}{|c|}{ Vitamin D3 level } \\
\hline & 29 & 16.7 & & 70 & 39.8 \\
\hline Grade I & \multicolumn{5}{|c|}{ Normal } \\
\hline Grade II & 105 & 59.7 & Insufficiency & 50 & 28.4 \\
\hline Grade III & 42 & 23.9 & Deficiency & 56 & 31.8 \\
\hline Site & \multicolumn{5}{|c|}{ CA 19-9 } \\
\hline Head & 75 & 42.6 & Mean \pm SD & \multicolumn{2}{|c|}{$1011.46 \pm 1149.19$} \\
\hline Body & 57 & 32.4 & Median (Range) & \multicolumn{2}{|c|}{$698(21-7896)$} \\
\hline Tail & 44 & 25 & & & \\
\hline \multirow[t]{2}{*}{ Peritoneal nodules } & \multirow[t]{2}{*}{109} & \multirow[t]{2}{*}{61.9} & $\begin{array}{l}\text { Response } \\
\text { PR }\end{array}$ & 50 & 28.4 \\
\hline & & & SD & 48 & 27.3 \\
\hline Liver metastasis & 135 & 76.7 & PD & 78 & 44.3 \\
\hline Lung metastasis & 54 & 30.7 & Follow-up (months) Mean \pm SD & & \\
\hline Bone & 3 & 1.7 & Median (Range) & & 60) \\
\hline $\begin{array}{l}\text { Treatment type } \\
\text { Chemotherapy }\end{array}$ & 146 & 83 & & & \\
\hline Best supportive care & 30 & 17 & & & \\
\hline
\end{tabular}

ECOG, Eastern Cooperative Oncology Group Performance Status.

$\mathrm{PR}$, partial response; SD, stable disease; PD, progressive disease.

Categorical variables were expressed as number (percentage).

Continuous variables were expressed as mean \pm SD \& median (range).

vitamin $D$ levels were detected in $60.2 \%$ of patients (insufficiency and deficiency were $28.4 \%$ and $31.8 \%$, respectively) (Table 1 ).

\subsection{The relation between the clinicopathological features, outcome, and vitamin D levels}

Lower vitamin D levels were positively associated with older age, larger tumor size, a higher grade of adenocarcinoma, higher serum level of CA 19-9, the presence of metastasis either to the liver or bones, poor ECOG PS, and low overall response rate (ORR) $(p<0.001)$. But there were no statistically significant differences between normal and lower vitamin D serum levels, either insufficiency or deficiency, in terms of sex, tumor site, and metastasis either to the lungs or peritoneum (Table 2).

\subsection{The relation between vitamin $\mathrm{D}$ levels and OS}

Unfortunately, all of the studied cases died during the follow-up period. The median follow-up period was 7.6 months (range 0.6-18.6). The ORR was $23.2 \%$, $54 \%$, and $82.9 \%$ for patients with vitamin D deficiency, insufficiency, and normal level of vitamin D, respectively. The median OS was 7.6 months for the whole group. The median OS for patients with normal level of vitamin D was 11.4 months, compared to 7.03 months for patients with vitamin $D$ insufficiency and 2.7 months for patients with vitamin D deficiency (Figure 1A, B). 
Table 2: Relation between clinicopathological features, outcome and vitamin D3 level among 176 pancreatic carcinoma patients

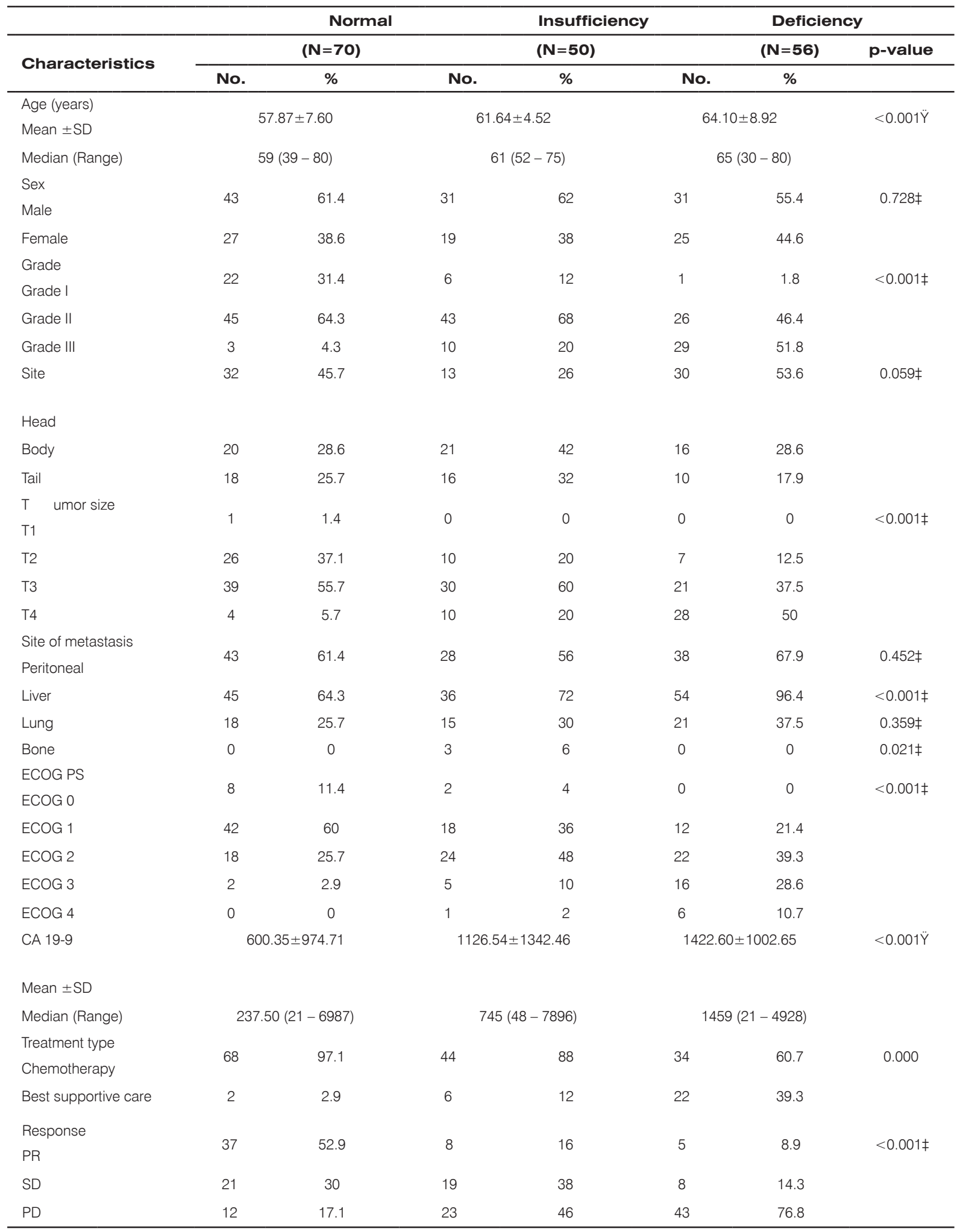

ECOG, Eastern Cooperative Oncology Group Performance Status.

$\mathrm{PR}$, partial response; $\mathrm{SD}$, stable disease; $\mathrm{PD}$, progressive disease

Continuous variables were expressed as mean \pm SD \& median (range); Categorical variables were expressed as number (percentage); Kruskal Wallis $\mathrm{H}$ test; $\neq$ Chi-square test; $\mathrm{p}<0.05$ is significant. 
Table 3: Multivariate cox regression hazard model for factors affecting cancer-specific survival in patients with APC.

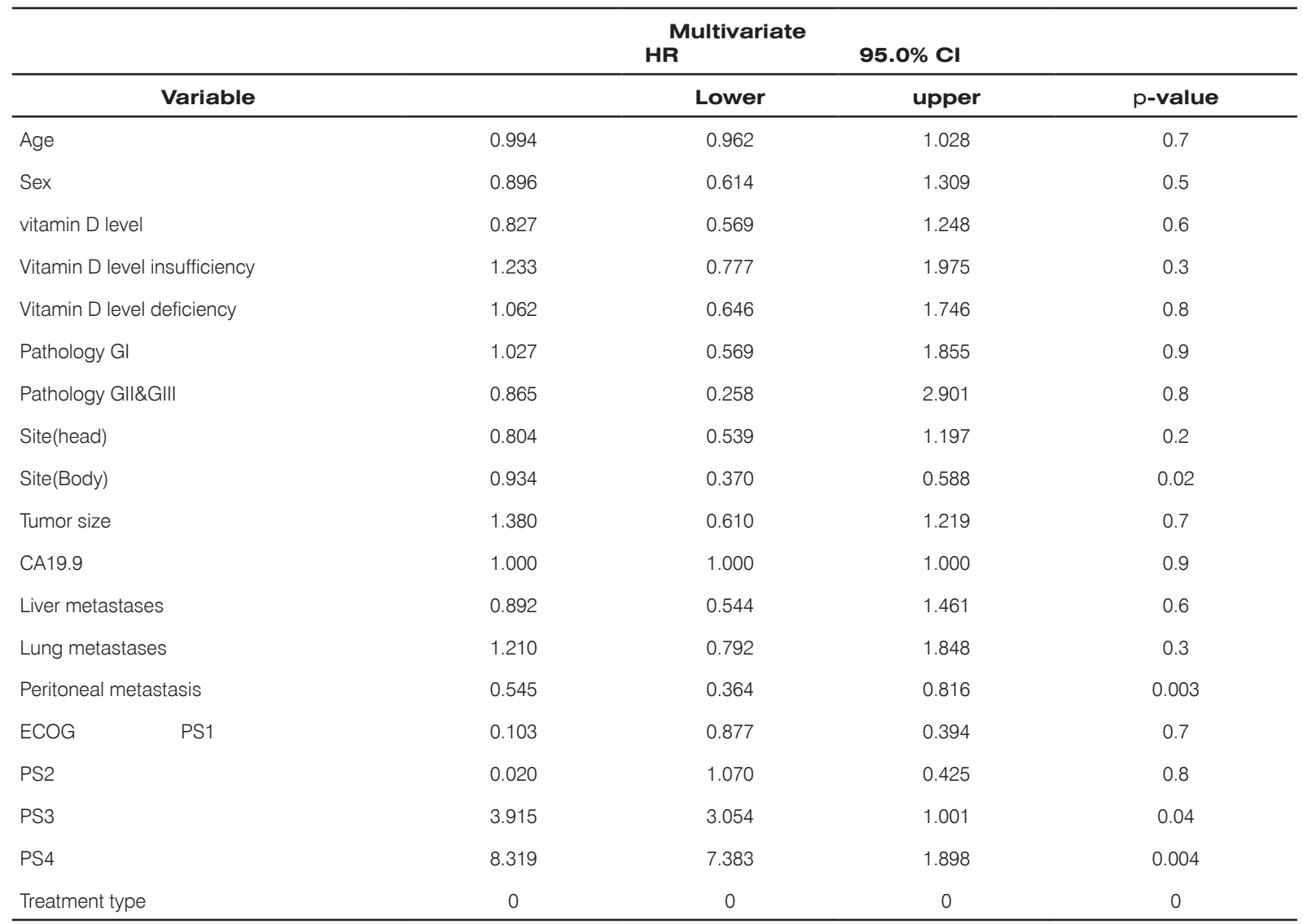

ECOG, Eastern Cooperative Oncology Group Performance Status.

The multivariate cox regression hazard model for treatment type is zero, because of constant or linearly dependent covariates (the degree of freedom reduced).

Table 4: Relation between vitamin D3 level and overall survival among 176 pancreatic carcinoma patients

\begin{tabular}{|c|c|c|c|c|c|c|c|c|c|}
\hline \multirow[b]{3}{*}{ All patients } & \multirow{3}{*}{$\begin{array}{c}\mathbf{N} \\
176\end{array}$} & \multirow{2}{*}{\multicolumn{2}{|c|}{$\begin{array}{c}\text { Mean OS (months) } \\
\text { Estimate }(95 \% \mathrm{Cl})\end{array}$}} & \multirow{2}{*}{\multicolumn{2}{|c|}{$\begin{array}{c}\text { Median OS (months) } \\
\text { Estimate }(95 \% \mathrm{Cl})\end{array}$}} & \multicolumn{3}{|c|}{ Overall Survival (OS) } & \multirow{3}{*}{ p-value } \\
\hline & & & & & & \multirow{2}{*}{$\frac{\text { 6month }}{55.7 \%}$} & \multicolumn{2}{|c|}{12 month 18 month } & \\
\hline & & 7.72mon & $(6.95-8.48)$ & 7.60mon & $(5.60-9.59)$ & & $26.1 \%$ & $0 \%$ & \\
\hline $\begin{array}{c}\text { Vitamin D3 level } \\
\text { Normal }\end{array}$ & 70 & $10.57 \mathrm{mon}$ & $(9.48-11.65)$ & ) $11.36 \mathrm{mon}$ & $(10.10-12.63)$ & $78.6 \%$ & $44.3 \%$ & $0 \%$ & $<0.001$ \\
\hline Insufficiency & 50 & $6.77 \mathrm{mon}$ & $(5.48-8.06)$ & 7.03mon & $(5.10-8.95)$ & $56 \%$ & $16 \%$ & $0 \%$ & \\
\hline Deficiency & 56 & $5.01 \mathrm{mon}$ & $(3.82-6.20)$ & $2.66 \mathrm{mon}$ & $(2.14-3.19)$ & $26.8 \%$ & $12.5 \%$ & $0 \%$ & \\
\hline
\end{tabular}

Continuous variables were expressed as mean $(95 \% \mathrm{Cl})$; categorical variables were expressed as number (percentage); $†$ Log rank test; $p<0.05$ is significant. 


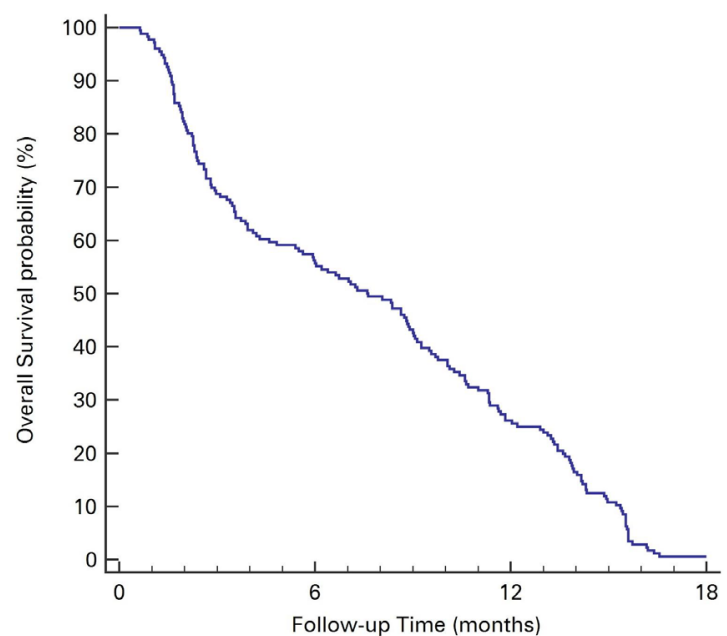

(B)

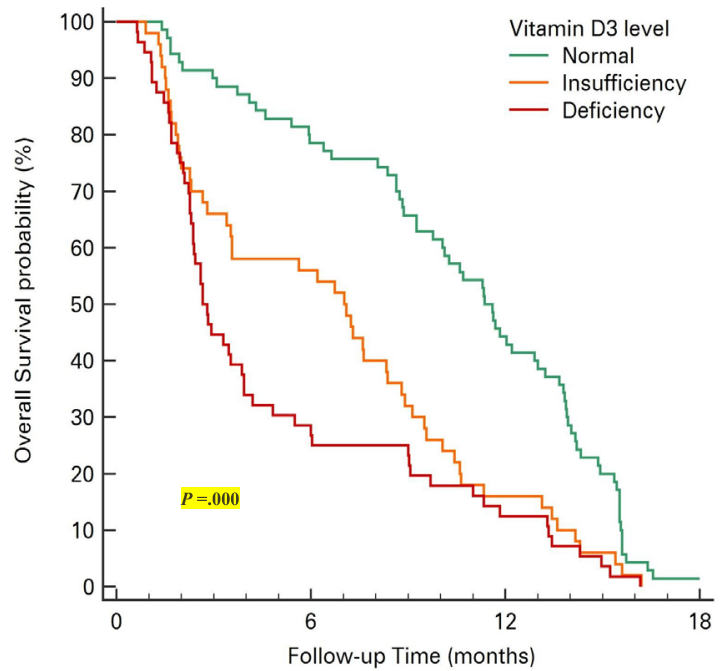

Figure 1: A: Kaplan-Meier survival curve for all studied patients. B: Survival analysis stratified by serum vitamin D3 levels.

\subsection{Multivariate and univariate Cox regression hazard model for factors affecting survival}

Analysis using the univariate Cox proportional hazards model showed that low vitamin D levels were associated with poor outcome. For vitamin D insufficiency: regression coefficient, 0.680; standard error, 0.190; Hazards Ratio (HR), 1.974 (95\% Cl: 1.362-2.862, <0.001), and for vitamin D deficiency: regression coefficient, 0.979; standard error, 0.186; HR, 2.661 (95\% Cl: 1.362$2.862,<0.001)$. Moreover, this statistically significant correlation continued with age, pathology type, CA 19-9 level, presence of metastasis, ECOG PS, and treatment type. In contrast, these significant relationships were lost in multivariate analysis (Table 3 ).

The OS at 6 months was $78.6 \%, 56 \%$, and $26.9 \%$ and at 12 months was $44.3 \%, 16 \%$, and $12.5 \%$ for patients with normal, insufficient, and deficient serum vitamin D levels, respectively (Table 4).

\section{Discussion}

Although significant advances have been made in the molecular biology of PC, their reflection on the treatment or diagnosis is not satisfactory. As the mortality rate is more or less equal to the incidence rate, APC represents a dismal prognosis for family, patients, and even oncologists. Worldwide, APC remains a major health burden. Most of the patients present in an advanced stage, while the treatment is given with palliative intent ${ }^{[14]}$.
Our results showed an association of low serum levels of vitamin $D$, either insufficiency or deficiency, with female sex, older age, larger tumor size, higher tumor grade, higher serum level of CA 19-9, presence of metastasis, poor ECOG PS, low ORR, and OS ( $p<$ $0.001)$. These findings are consistent with the findings from CALGB 80303 (Alliance) on 256 patients with APC evaluating 25(OH)D levels and outcome ${ }^{[15]}$. The association of lower vitamin $D$ serum levels with APC may be related to the general debilitating condition in case of severe anorexia, poor oral intake (dietary sources), and the lack of sun exposure (more than $90 \%$ of vitamin D status derived from sunlight exposure). Although our country has a sunny weather, lower vitamin $D$ levels are not uncommon. The lifestyle and the traditional conservative long Egyptian dress with the hijab, as well as our cultural practice do not advice people to spend long time outdoors. Moreover, in the univariate Cox regression hazard model, these lower levels were associated with poor outcome $(p<0.001)$. These results are matching with many previous studies ${ }^{[16-26]}$. On multivariate analysis, a positive association was found only with peritoneal metastasis and ECOG PS. Due to the constant or linearly dependent covariates, the degree of freedom reduced, so the correlation with the treatment type was not detectable.

In 2006, Giovannucci et al. conducted the first prospective trial evaluating the correlation between serum vitamin D levels and cancer mortality. The final report showed a statistically significant inverse association of low serum levels of vitamin D and PCrelated death ${ }^{[16]}$. 
Later on, five studies were conducted to evaluate the relationship between serum vitamin $D$ levels and PC-related mortality ${ }^{[17-21]}$. In the subgroup analysis, high serum levels of vitamin $D$ were associated with a favorable outcome of APC, although it did not reach a statistically significant level. Moreover, Tretli et al. reported that higher serum $D$ levels were associated with survival improvement in a variety of cancers including $\mathrm{PC}^{[22]}$. A meta-analysis review including 853 studies reported that high serum levels of vitamin $D$ were associated with improved outcome ${ }^{[23]}$.

In another meta-analysis of standardized vitamin D in 26,916 individuals, Gaksch et al. showed an association between low vitamin D levels and unfavorable outcome ${ }^{24]}$.

Although most of the studies proved the correlation between vitamin $D$ insufficiency or deficiency and poor outcome, there are some studies contradicting these observations. A correlative, national, randomized, double-blind, multicenter, Phase III study showed a lack of statistically significant correlation between the baseline levels of vitamin D and OS or PFS in patients with $\mathrm{APC}^{[15]}$.

The mechanisms by which vitamin $D$ exerts its anticancer effects are not clearly defined. The implication in gene control responsible for cellular proliferation, differentiation, apoptosis, and immune modulation may be the principle. Gene activation occurs mainly through two pathways: the first one is the classical vitamin D/ VDR pathway, which is necessary for the subsequent mobilization of coactivators and more gene activation; the second one is the non-classical vitamin D/VDR pathway wherein it occurs through the interaction with the wnt-/ $\beta$-catenin/TCF pathway. Moreover, vitamin $D$ is considered as a stromal-depleting agent due to its antiinflammatory property resulting in a reduction in fibrosis, and promotes what is called stromal remodeling, which leads to an increase in intratumor chemotherapy perfusion ${ }^{[25]}$.

This proposed antitumor activity of vitamin $D$ against PC has driven widespread interest in evaluating its therapeutic effect, either alone or in combination with chemotherapy. VITdCUT is a recruiting prospective Phase III trial evaluating the value of oral vitamin D supplementation in altered serum vitamin $D$ levels in PC. The trial aims to compare higher doses versus a standard dose of vitamin $D$ in these subtypes of patients. However, still, the results are pending; if positive results are obtained, vitamin $\mathrm{D}$ may be incorporated as a standard supportive of care in PC (ClinicalTrials.gov Identifier: NCT03472833).

Given the role of dietary intake and PC risk, analytical epidemiological studies revealed conflicting results ${ }^{[26]}$.
Two important prospective analyses were done in the Health Professionals Follow-up Study (HPFS) and Nurses' Health Study (NHS) and they reported the possibility of a protective role of vitamin D in PC. The first analysis conducted in the HPFS showed that higher vitamin $D$ scores were associated with a decrease in both the incidence and mortality of $\mathrm{PC}{ }^{[16]}$. The other one was an analysis conducted by both the HPFS and NHS, which reported an inverse association between vitamin D supplementation and $\mathrm{PC}{ }^{[27]}$.

Unlike most of the studies that had shown an inverse relation between serum vitamin $D$ levels and PC risk, a nested case-control analysis done in the AlphaTocopherol, Beta-Carotene Cancer Prevention Study (ATBC) that included 200 male Finnish smokers with PC and 400 controls found an approximately threefold increased risk in patients in the highest compared with the lowest quintile of vitamin D serum levels (OR 2.92; $95 \% \mathrm{Cl} 1.56-5.48)^{[28]}$. However, the same investigator group and a more recent research using a different group population failed to assert this positive relationship (OR 1.45; Cl 0.66-3.15; $p=0.49)^{[29]}$. In another pooled analysis from PC case-control consortium, the researchers reported that the cancer risk increased with higher dietary vitamin $D$ intake, although they did not deny the beneficial effect of vitamin $D$ obtained from exposure to ultraviolet light ${ }^{[8]}$.

Owing to these conflicting data and the paucity of other results, there is no clear value to recommend serum vitamin $\mathrm{D}$ evaluation for treatment of $\mathrm{PC}$.

\section{Limitations}

Although this is a prospective trial, small sample size and short duration of follow-up, in addition to the inability to use the international guidelines in the treatment of APC due to financial issues represented the main limitations of this study.

Our country is considered a developing country with a low economic status. Most of the patients with PS are diagnosed at an advanced stage. Even for those patients diagnosed with PS at an early stage, there is some delay to get proper treatment as not all our patients covered by health insurance. Besides, there is lack of supportive treatment.

Furthermore, seasonal variations in the measurement of serum vitamin $D$ levels were not considered (winter months differ from sunny months). So, a single measurement may not be enough as it may be affected by the time of blood sampling. 


\section{Conclusion and recommendation Conflict of interest}

Many data including our results suggest an inverse relation of the serum levels of vitamin $D$ and outcome in patients with APC.

Currently, there is no strong clinical evidence to make healthcare providers advice regular vitamin $D$ supplementation to treat or decrease the risk of PC. None has proven etiology and effect.

Owing to the proposed multiple antitumor mechanisms, many trials are ongoing to evaluate the therapeutic role of vitamin D or its analogs in the management of PC. Longer and larger multicenter prospective trials are needed.

\section{References}

[1] Siegel RL, Miller KD, Jemal A. CA Cancer J Clin. 2018; 68:7-30.

[2] Rahib L, Smith BD, Aizenberg R, Rosenzweig AB, Fleshman JM, Matrisian LM. Cancer Res. 2014 ; 74 : 2913-2921.

[3] American Cancer Society. Cancer facts \& figures 2017.

[4] Van der Rhee H, Coebergh JW, de Vries E. Is prevention of cancer by sun exposure more than just the effect of vitamin D? A systematic review of epidemiological studies. Eur J Cancer. 2013; 49:1422-1436.

[5] Ng K, Sargent DJ, Goldberg RM, Meyerhardt JA, Green EM, Pitot HC, et al. Vitamin D status in patients with stage IV colorectal cancer: findings from Intergroup trial N9741. J Clin Oncol. 2011; 29:1599-1606.

[6] Holick MF. Vitamin D deficiency. N Engl J Med, 2007; 357, 266-281.

[7] Jacobs ET, Kohler LN, Kunihiro AG, Jurutka PW. Vitamin $D$ and colorectal, breast, and prostate cancers: A review of the epidemiological evidence. J Cancer, 2016; 7, 232-240.

[8] Waterhouse M, Risch HA, Bosetti C, Anderson KE, Petersen GM, Bamlet WR, et al. Vitamin D and pancreatic cancer: a pooled analysis from the pancreatic cancer case-control consortium. Ann Oncol 2015; 26:1776-83.10.1093.

[9] Moukayed M, Grant WB. Molecular link between vitamin D and cancer prevention. Nutrients. 2013; 5:3993-4021.10.3390/nu5103993.

[10] Jorde R, Grimnes G. Vitamin D and health: the need for more randomized controlled trials. J Steroid
The authors certify that there is no potential or actual conflict of interest related to this research.

Biochem Mol Biol. 2015; 148:269-74.10.1016.

[11] Avenell A, MacLennan GS, Jenkinson DJ, McPherson GC, McDonald AM, Pant PR, et al. Long-term follow-up for mortality and cancer in a randomized placebo-controlled trial of vitamin $\mathrm{D}$ (3) and/or calcium (RECORD trial). J Clin Endocrinol Metab. 2012; 97:614-22.10.1210.

[12] Grant WB. A Review of the Evidence Supporting the Vitamin D Cancer Prevention Hypothesis in 2017. Anticancer Res. 2018; 38:1121-1136.

[13] Gallagher JC, Sai AJ. Vitamin D insufficiency, deficiency, and bone health. J Clin Endocrinol Metab. 2010; 95:2630-2633.

[14] Cannon TL, Ford J, Hester D, Trump DL. The Incidental Use of High-Dose Vitamin D3 in Pancreatic Cancer. Case Reports in Pancreatic Cancer Volume 2.1, 2016

[15] Van Loon K, Owzar K, Jiang C, Kindler HL, Mulcahy MF, Niedzwiecki D, O'Reilly EM, Fuchs C, Innocenti F, Venook AP; Alliance for Clinical Trials in Oncology.25-Hydroxyvitamin D levels and survival in advanced pancreatic cancer: findings from CALGB 80303 (Alliance). J Natl Cancer Inst. 2014: 6;106.

[16] Giovannucci E, Liu Y, Rimm EB, Hollis BW, Fuchs CS, Stampfer MJ, et al. Prospective Study of Predictors of Vitamin D Status and Cancer Incidence and Mortality in Men J Natl Cancer Inst. $2006 ; 5 ; 98: 451-459$.

[17] Cho M, Peddi PF, Ding K, Chen L, Thomas D, Wang J, et al. Vitamin D deficiency and prognostics among patients with pancreatic adenocarcinoma. J Transl Med. 2013; 11:206. 
[18] Haas M, Kern C, Kruger S, Michl M, Modest DP, Giessen $C$, et al. Assessing novel prognostic serum biomarkers in advanced pancreatic cancer: the role of CYFRA 21-1, serum amyloid $A$, haptoglobin, and 25-OH vitamin D3. Tumour Biol. 2015; 36:26312640.

[19] McGovern EM, Lewis ME, Niesley ML, Huynh N, Hoag JB. Retrospective analysis of the influence of 25-hydroxyvitamin $\mathrm{D}$ on disease progression and survival in pancreatic cancer. Nutr J. 2016; 15:17.

[20] Van Loon K, Owzar K, Jiang C, Kindler HL, Mulcahy MF, Niedzwiecki D, et al. 25-Hydroxy vitamin D levels and survival in advanced pancreatic cancer: findings from CALGB 80303 (Alliance). J Natl Cancer Inst. 2014; 106.

[21] Yuan C, Qian ZR, Babic A, Morales-Oyarvide V, Rubinson DA, Kraft P, et al. Prediagnostic plasma 25-hydroxyvitamin $D$ and pancreatic cancer survival. J Clin Oncol. 2016; 34:2899-2905.

[22] Tretli S, Schwartz GG, Torjesen PA, Robsahm TE. Serum levels of 25-hydroxyvitamin $D$ and survival in Norwegian patients with cancer of breast, colon, lung, and lymphoma: a population-based study. Cancer Causes Control. 2012; 23:363-370.

[23] Zhang X, Huang XZ, Chen WJ, Wu J, Chen Y, Wu CC, et al. Plasma 25-hydroxyvitamin D levels, vitamin D intake, and pancreatic cancer risk or mortality: a meta-analysis. Oncotarget. 2017: 29; 8:64395-64406

[24] Gaksch M, Jorde R, Grimnes G, Joakimsen R, Schirmer $H$, Wilsgaard $T$, et al. Vitamin $D$ and mortality: individual participant data meta-analysis of standardized 25-hydroxyvitamin D in 26916 individuals from a European consortium. PLoS One. 2017; 12: e0170791.

[25] Duffy MJ, Murray A, Synnott NC, O'Donovan N, Crown J. Vitamin D analogues: Potential use in cancer treatment. Crit Rev Oncol Hematol. 2017; 112:190-197

[26] Van Duijnhoven FJB, Jenab M, Hveem K, Siersema PD, Fedirko V, Duell EJ, et al. Circulating concentrations of vitamin $D$ in relation to pancreatic cancer risk in European populations. Int $\mathrm{J}$ Cancer. 2018; 15; 142:1189-1201.

[27] Skinner HG, Michaud DS, Giovannucci E, Willett WC, Colditz GA, Fuchs CS. Vitamin D intake and the risk for pancreatic cancer in two cohort studies. Cancer Epidemiol Biomarkers Prev 2006; 15:16881695.

[28] Stolzenberg-Solomon RZ, Vieth R, Azad A, et al. A prospective nested case-control study of vitamin $D$ status and pancreatic cancer risk in male smokers. Cancer Res. 2006; 66:10213-10219.
[29] Stolzenberg-Solomon RZ, Hayes RB, Horst RL, et al. Serum vitamin $D$ and risk of pancreatic cancer in the prostate, lung, colorectal, and ovarian screening trial. Cancer Res. 2009; 69:1439-1447. 